\title{
Anti-inflammatory effects of low molecular weight heparin derivative in a rat model of carrageenan-induced pleurisy
}

\author{
Matteo Ceccarelli ${ }^{a}$, Daniele Bani ${ }^{b}$, Lorenzo Cinci ${ }^{b}$, Silvia Nistri ${ }^{b}$, Caterina Uliva ${ }^{a}$, Elena Ragazzo ${ }^{a}$, \\ Alfredo Vannacci ${ }^{a}$, Marco Manoni ${ }^{d}$, Anna Maria Gori ${ }^{c, e}$, Rosanna Abbate ${ }^{\text {c, }}$ \\ Gian Franco Gensini ${ }^{c}$, Emanuela Masini ${ }^{a}$, * \\ ${ }^{a}$ Department of Preclinical and Clinical Pharmacology, University of Florence, Florence, Italy \\ ${ }^{b}$ Department of Anatomy, Histology and Forensic Medicine, Sect. Histology, University of Florence, Florence, Italy \\ ${ }^{c}$ Department of Medical and Surgical Critical Care, Section of Clinical Medicine and Cardiology, University of Florence, Florence, Italy \\ ${ }^{d}$ INALCO RSM S.p.A., Research Center, Montale, Pistoia, Italy \\ ${ }^{e}$ Centro S. Maria agli ulioi, Fondazione Don Carlo Gnocchi, Onlus IRCCS, Florence, Italy
}

Received: July 29, 2008; Accepted: December 30, 2008

\section{Summary}

Low molecular weight heparin derivatives are characterized by low anti-coagulant activity and marked anti-inflammatory effects that allow for these molecules to be viewed as a new class of non-steroidal anti-inflammatory drugs (NSAIDs). We show here that K5NOSepiLMW, an 0-sulphated heparin-like semi-synthetic polymer of the D-glucuronic acid-N-acetyleparoson disaccharide unit with low molecular weight, has marked anti-inflammatory effects in a rat model of acute inflammation, the carrageenan-induced pleurisy, commonly used to test NSAID efficacy. A 30-min. pre-treatment with K5NOSepiLMW $(0.1,0.5$ and $1 \mathrm{mg} / \mathrm{kg}$ b.wt., given intrapleurally) attenuated the recruitment of leucocytes in the lung tissue and the pleural exudate, inhibited the induction of inducible nitric oxide synthase and cyclooxygenase-2 (COX-2), thereby abating the generation of nitric oxide and pro-inflammatory prostaglandins such as $\mathrm{PgE}_{2}$ and $\mathrm{PGF}_{1 \alpha}$, reduced the inflammation-induced nitroxidative lung tissue injury, as shown by tissue thiobarbituric acid-reactive substances and nitrotyrosine, and blunted the local generation of cytokines such as interleukin- $1 \beta$ and tumour necrosis factor- $\alpha$. All these parameters were markedly increased by intrapleural carrageenan in the absence of any pre-treatment. The anti-inflammatory action of K5NOSepiLMW is specific, as judged by the lack of therapeutic effects of B4/110, a biologically inactive cognate polysaccharide, given in the place of the authentic molecule. Moreover, K5NOSepiLMW showed similar effects as celecoxib (1 mg/kg b.wt), a COX-2 inhibitor and well-known NSAID. This study provides further insight into the mechanisms underlying the beneficial effects of heparin derivatives in inflammation and identifies K5NOSepiLMW as a novel, promising anti-inflammatory drug.

Keywords: low molecular weight heparin $\bullet$ nitric oxide synthase $\bullet$ cyclooxygenase-2 $\bullet$ leucocytes $\bullet$ cytokines $\bullet$ pleurisy

\section{Introduction}

The major hallmark of inflammation is the recruitment and activation of leucocytes, especially polymorphonuclear neutrophils (PMNs), which results from a complex interplay among these cells and the vascular endothelium [1-3] and accounts for destruction of foreign pathogens on one hand, and breakdown and remodelling of inflamed tissues on the other hand [4]. Inflammation is trig-

\footnotetext{
*Correspondence to: Prof. Emanuela MASINI, M.D.,

Department of Preclinical and Clinical Pharmacology,

University of Florence, Viale G. Pieraccini 6, 50139 Florence, Italy.

Tel.: + 390554271233

Fax: +390554271280

E-mail: emanuela.masini@unifi.it
}

gered and sustained by pro-inflammatory cytokines as well as prostaglandins and gaseous radicals, such as nitric oxide [4-6], which are produced locally by the inflammation-induced enzymes cyclooxygenase-2 (COX-2) and nitric oxide synthase (iNOS). Most of the classical anti-inflammatory drugs target the endogenous production of these pro-inflammatory mediators.

In this context, the sulphated proteoglycan heparin can be also regarded as a non-conventional anti-inflammatory molecule, as it has several biological actions independent of its well-known anticoagulant activity, including the modulation of extracellular matrix synthesis, cell proliferation, angiogenesis and inflammation [7-11]. In particular, heparin has been shown to inhibit proinflammatory cytokine production by human monocytes stimulated 
by lipopolysaccharide or interferon- $\gamma$ [12]. On these grounds, in recent years, considerable efforts have been made to identify the molecular domains of heparin responsible for its anti-inflammatory properties and to develop synthetic derivatives with low or no anti-coagulant properties and clear-cut anti-inflammatory effects [13]. In animal models of inflammation, such as acute peritonitis and organ-specific ischemia reperfusion, these anti-inflammatory heparin-like molecules effectively inhibited leucocyte adhesion and migration [13].

We have recently studied the anti-inflammatory properties of K5 polysaccharide obtained from Escherichia coli strain 010:K5:H4. This is a polymer of the D-glucuronic acid and $\mathrm{N}$-acetyleparoson disaccharide unit, the precursor of heparin and heparan sulphate. This structural affinity makes the K5 polysaccharide an optimal precursor for the synthesis of sulphated heparin semi-synthetic derivatives. In particular, the low molecular weight compound K5-N, OS epi (K5NOSepiLMW) lacks appreciable anti-thrombotic activity and is able to inhibit the production of pro-inflammatory cytokines from human lipopolysaccharide-stimulated mononuclear cells, without influencing other anti-inflammatory cytokines such as interleukin (IL)-10 [14]. The aim of the present study is to evaluate the effects of K5NOSepiLMW in a widely used in vivo model of acute inflammation in the rat, namely carrageenan-induced pleurisy, whose cellular and molecular mechanisms are well characterized [15-21].

\section{Materials and methods}

\section{Materials}

Primary rabbit polyclonal anti-nitrotyrosine (NT) antiserum, biotin-conjugated goat anti-rabbit IgG, and avidin-biotin peroxidase complex were obtained from Upstate Biotech (DBA, Milan, Italy). Rabbit polyclonal anti-COX-2 and anti-iNOS antisera were from Cayman Chemicals (Ann Arbor, MI, USA). Celecoxib was from LC Laboratories (Woburn, MA, USA). Unless otherwise stated, all other reagents and compounds used were obtained from Sigma (Milan, Italy). The semi-synthetic low molecular weight heparin polysaccharide K5-N,0Sepi (K5NOSepiLMW) was provided by INALCO RSM Research Center, Montale, Italy, as reported previously [14].

\section{Carrageenan-induced pleurisy and drug treatments}

Male Wistar albino rats (Charles River, Milan, Italy), weighing 250-300 g, were housed in a controlled environment and provided with standard rodent chow and water. The experimental protocols were designed in compliance with the Italian and the European Community regulations on animal experimentation for scientific purposes (D.M. 116192; O.J. of E.C. L358/1 12/18/1986).

Rats were anaesthetized with sodium pentobarbital (Abbott, Latina, Italy; $50 \mathrm{mg} / \mathrm{kg}$ b.wt., intraperitoneally) and submitted to a skin incision at the sixth left intercostal space to access the pleural cavity. The animals were then treated as described below.

Briefly, 48 rats were divided in seven groups, eight animals each:

(1) Sham-operated control animals, injected intrapleurally with $0.2 \mathrm{ml}$ sterile saline;

(2) Carrageenan-treated animals, injected intrapleurally with $\lambda$-carrageenan, $1 \% \mathrm{w} / \mathrm{v}$ in $0.2 \mathrm{ml}$ sterile saline;

(3)-(5) Animals pre-treated with K5NOSepiLMW, given intrapleurally at the doses of $0.1,0.5$ or $1 \mathrm{mg} / \mathrm{kg}$ b.wt. $30 \mathrm{~min}$. before carrageenan;

(6) Animals treated with B4/110, a biologically inactive non-sulphated polysaccharide compound used as negative control molecule, given intrapleurally at the dose of $1 \mathrm{mg} / \mathrm{kg}$ b.wt. $30 \mathrm{~min}$. before carrageenan;

(7) Animals treated with celecoxib, a COX-2 inhibitor used as reference anti-inflammatory drug, given intrapleurally at the dose of $1 \mathrm{mg} / \mathrm{kg}$ b.wt. 30 min. before carrageenan. This dose was consistent with that required to abrogate COX-2 activity [19].

After the injections, the skin incision was closed with a suture. Four hours later, the animals were killed with a lethal dose of sodium pentobarbital. The chest was carefully opened and the pleural cavity was rinsed with $2 \mathrm{ml}$ saline containing heparin $(5 \mathrm{IU} / \mathrm{ml})$ and indomethacin $(10 \mu \mathrm{g} / \mathrm{ml})$. The total volume of exudate and washing solution was collected. The exudates contaminated with blood were discarded. The amount of exudate was calculated by subtracting the volume injected $(2 \mathrm{ml})$ from the total volume recovered. Leucocytes in the exudate were stained with the vital dye Trypan Blue and counted with a Bürker chamber. Lung tissue specimens were also collected and immediately processed as described below.

\section{Evaluation of iNOS and COX-2 expression by Western blot analysis}

Lung tissue fragments were quickly minced and homogenized in $500 \mu \mathrm{l}$ cold lysis buffer containing $10 \mathrm{mmol} / \mathrm{l}$ Tris/ $\mathrm{HCl} \mathrm{pH} \mathrm{7.4,10} \mathrm{mmol/l} \mathrm{NaCl,}$ $1.5 \mathrm{mmol} / / \mathrm{MgCl} 2,2 \mathrm{mmol} / \mathrm{l} \mathrm{Na} 2$ ethylenediaminetetraacetic acid (EDTA), $1 \% \mathrm{v} / \mathrm{v}$ Triton X-100, $1 \mathrm{mmol} / \mathrm{l}$ phenylmethylsulfonyl fluoride, $20 \mu \mathrm{g} / \mathrm{ml}$ leupeptin, $1 \mu \mathrm{g} / \mathrm{ml}$ pepstatin, $1 \mathrm{mg} / \mathrm{ml}$ pefabloc and $2.5 \mu \mathrm{g} / \mathrm{ml}$ aprotinin. Upon centrifugation at $13,000 \times g$ at $4^{\circ} \mathrm{C}$ for 10 min., the supernatants were collected and the total protein content was measured spectrophotometrically using micro BCA Protein Assay Reagent Kit (Pierce, IL, USA). The samples, each containing $100 \mu \mathrm{g}$ of total proteins, were electrophoresed by SDS-PAGE (200 V, $1 \mathrm{hr}$ ) using a denaturating $7.6 \% \mathrm{v} / \mathrm{v}$ polyacrylamide gel and blotted onto nitrocellulose membranes (Amersham, Buckinghamshire, UK) (150 V, 1 hr). After thorough washings in PBS containing $0.1 \% \mathrm{v} / \mathrm{v}$ Tween (T-PBS), the membranes were blocked with T-PBS containing $5 \% \mathrm{w} / \mathrm{v}$ bovine serum albumin at room temperature for $1 \mathrm{hr}$ and incubated overnight at $4^{\circ} \mathrm{C}$ with rabbit polyclonal antisera against iNOS and COX-2, both diluted 1:1000 in T-PBS containing $1 \% \mathrm{w} / \mathrm{v}$ bovine serum albumin. After washing in T-PBS the membranes were incubated with peroxidase-labelled goat anti-rabbit antibody (Vector, Burlingame, CA, USA), diluted $1: 10,000$ in T-PBS containing $1 \% \mathrm{w} / \mathrm{V}$ bovine serum albumin, for $1 \mathrm{hr}$ at room temperature. Immune reaction was revealed by incubation with the enhanced chemiluminescent (ECC) substrate (Amersham) followed by exposure to high sensitivity photographic film (Biomax ML, Kodak, Rochester, NY, USA). As internal control, membranes immunostained with rabbit polyclonal anti $\beta$-actin antibody $(1: 20,000)$ were used. For iNOS and COX-2, quantitative evaluations of the band intensities were performed by computer-assisted densitometry, 
using the Scion Image Beta 4.0.2 image analysis program (Scion, Frederick, MD, USA). Each band was then normalized to that of actin.

\section{Determination of nitric oxide synthase activity}

The calcium-independent conversion of L-arginine to L-citrulline was assumed as iNOS activity, according to Cuzzocrea et al. [21]. Lung tissue specimens were homogenized in a buffer composed of: $50 \mathrm{mmol} / \mathrm{I} \mathrm{Tris}-\mathrm{HCl}, 0.1 \mathrm{mmol} / \mathrm{I} \mathrm{EDTA}$, and $1 \mathrm{mmol} / /$ phenylmethylsulfonyl fluoride, $\mathrm{pH} 7.4$, and then used for the $\mathrm{L}-\left[{ }^{3} \mathrm{H}\right]$ arginine to $\mathrm{L}-\left[{ }^{3} \mathrm{H}\right]$ citrulline conversion assay. Briefly, $50 \mu \mathrm{l}$ of each sample were incubated in the presence of $10 \mu \mathrm{mol} / / \mathrm{L}-\left[{ }^{3} \mathrm{H}\right]$ arginine $(5 \mathrm{kBq}$ per tube), $1 \mathrm{mmol} / / \mathrm{NADPH}^{+}, 30 \mathrm{nmol} / \mathrm{l}$ calmodulin, $5 \mu \mathrm{mol} / /$ tetrahydrobiopterin, and $2 \mathrm{mmol} / \mathrm{l}$ ethylene glycol tetro-acetic acid (EGTA) for $20 \mathrm{~min}$. at $22^{\circ} \mathrm{C}$. The reaction was stopped by $0.5 \mathrm{ml}$ ice-cold HEPES buffer, $\mathrm{pH} 5.5$, containing $2 \mathrm{mmol} / \mathrm{I} \mathrm{EGTA}$ and $2 \mathrm{mmol} / \mathrm{I} \mathrm{EDTA}$. Reaction mixtures were applied to Dowex 50W ( $\mathrm{Na}^{+}$form) columns and the eluted L$\left[{ }^{3} \mathrm{H}\right]$ citrulline activity was measured by a scintillation counter (Beckman Coulter, Inc., Fullerton, CA, USA).

\section{Measurement of nitrites}

Total nitrites, the stable end products of nitric oxide, were measured in the exudate as described previously [18]. Briefly, nitrates in the sample were first reduced to nitrites by incubation with $280 \mathrm{mU} / \mathrm{ml}$ nitrate reductase and $160 \mu \mathrm{mol} / \mathrm{l} \mathrm{NADPH}{ }^{+}$at room temperature for $3 \mathrm{hrs}$. The total nitrite concentration in the samples was then measured using the Griess reaction by adding $100 \mu \mathrm{l}$ of Griess reagent $(0.1 \%$ w/v naphthylethylendiamide dihydrochloride in water and $1 \% \mathrm{w} / \mathrm{v}$ sulfanilamide in $5 \% \mathrm{v} / \mathrm{v}$ $\mathrm{H}_{3} \mathrm{PO}_{4}$ ) to $100 \mu \mathrm{l}$ of the samples. Optical density was measured at $550 \mathrm{~nm}$ wavelength using a microplate reader (SLT-Lab Instruments, Salzburg, Austria). Nitrite concentrations were calculated by comparison with a standard curve of sodium nitrite.

\section{Evaluation of cyclooxygenase activity}

Lung tissue specimens were homogenized in a buffer composed of: $20 \mathrm{mmol} / \mathrm{l} \mathrm{HEPES}$, pH 7.2, $320 \mathrm{mmol} / \mathrm{l}$ sucrose, $1 \mathrm{mmol} / \mathrm{l}$ dithiothreitol, $10 \mu \mathrm{g} / \mathrm{ml}$ styrosporin, $2 \mu \mathrm{g} / \mathrm{ml}$ aprotinin and $10 \mu \mathrm{g} / \mathrm{ml}$ leupeptin. The homogenates were incubated at $37^{\circ} \mathrm{C}$ for $30 \mathrm{~min}$. in the presence of excess arachidonic acid $(30 \mu \mathrm{mol} / \mathrm{l})$. The samples were boiled and centrifuged at $10,000 \times g$ for $5 \mathrm{~min}$. The concentration of 6 -keto-PGF1 $\alpha$ in the supernatant was then measured by radioimmunoassay as previously described [22]. Protein concentration in each homogenate was measured using the Bradford assay using a standard curve of bovine serum albumin [23].

\section{Myeloperoxidase (MP0) activity}

MPO activity, an indicator of PMN accumulation, was determined as described previously [24]. Lung tissue specimens were weighed and homogenized in a buffer composed of: $0.5 \% \mathrm{w} / \mathrm{v}$ hexadecyltrimethylammonium bromide dissolved in $10 \mathrm{mmol} / \mathrm{l}$ potassium phosphate buffer, pH 7.0, and centrifuged for $30 \mathrm{~min}$. at $20,000 \times g$ at $4^{\circ} \mathrm{C}$. An aliquot of the supernatant was then allowed to react with a solution of $1.6 \mathrm{mmol} / \mathrm{l}$ tetramethylbenzidine and $0.1 \mathrm{mmol} / \mathrm{l}$ hydrogen peroxide. The rate of change in absorbance was measured spectrophotometrically at $650 \mathrm{~nm}$ wavelength. MPO activity was defined as the quantity of enzyme degrading $1 \mu \mathrm{mol}$ of peroxide per min. at $37^{\circ} \mathrm{C}$ and was expressed in $\mathrm{mU} / \mathrm{g}$ wet tissue.

\section{Thiobarbituric acid-reactive substance (TBARS) measurement}

TBARS levels in the lung tissue, an indicator of lipid peroxidation, were determined as described previously [25]. Lung tissue specimens were homogenized in $1.15 \% \mathrm{w} / \mathrm{v} \mathrm{KCl}$ solution. A $100 \mu$ l aliquot of the homogenate was added to a reaction mixture containing $200 \mu \mathrm{l}$ of $8.1 \% \mathrm{~W} / \mathrm{v}$ SDS, $1.5 \mathrm{ml}$ of $20 \% \mathrm{v} / \mathrm{v}$ acetic acid, pH $3.5,1.5 \mathrm{ml}$ of $0.8 \% \mathrm{w} / \mathrm{v}$ thiobarbituric acid and $700 \mu \mathrm{l}$ of distilled water. Samples were then boiled for $1 \mathrm{hr}$ at $95^{\circ} \mathrm{C}$ and centrifuged at $3000 \times g$ for $10 \mathrm{~min}$. The absorbance of the supernatant was measured spectrophotometrically at $532 \mathrm{~nm}$ wavelength.

\section{Immunohistochemical localization of nitrotyrosine}

$\mathrm{NT}$, an index of protein nitrosylation by harmful oxidants generated during inflammation, such as peroxynitrite, was determined by immunohistochemistry as described previously [21]. Sections from formaldehyde-fixed, paraffin-embedded lung tissue specimens were rehydrated, endogenous peroxidase was quenched with $0.3 \% \mathrm{v} / \mathrm{v}$ hydrogen peroxide in $60 \% \mathrm{v} / \mathrm{v}$ methanol for $30 \mathrm{~min}$., the sections were permeabilized with $0.1 \% \mathrm{w} / \mathrm{v}$ Triton X-100 in PBS for 20 min., non-specific labelling was minimized by incubating the section in $2 \% \mathrm{v} / \mathrm{v}$ normal goat serum in PBS for $20 \mathrm{~min}$., the sections were incubated with rabbit polyclonal anti-NT antiserum (Upstate Biotechnology, Buckingham, UK; $1: 100$ ) at $4^{\circ} \mathrm{C}$ overnight, immune reaction was revealed by goat anti-rabbit IgG conjugated with biotin (Vector Lab, Burlingame, CA, USA; 1:200) followed by incubation with ABC complex (Vector Lab; 1:200). Negative controls were carried out by omitting the primary or the secondary antibodies. The sections were counterstained with haematoxylin before mounting.

\section{Evaluation of nitrotyrosine expression by dot blot analysis}

Samples of lung tissue homogenates, each containing $200 \mu \mathrm{g}$ of total proteins, were spotted on nitrocellulose membrane strips (Amersham). They were air-dried for $30 \mathrm{~min}$. and then saturated with T-PBS containing $5 \% \mathrm{w} / \mathrm{v}$ bovine serum albumin for $1 \mathrm{hr}$ at room temperature. The strips were subsequently incubated with rabbit polyclonal antiserum against NT (Upstate), diluted 1:300 in T-PBS containing 1\% w/v bovine serum albumin, for $1 \mathrm{hr}$ at room temperature. After washing in T-PBS the membranes were incubated with peroxidaselabelled goat anti-rabbit antibodies diluted 1:10,000 in T-PBS containing 1\% W/v bovine serum albumin, for $1 \mathrm{hr}$ at room temperature. Immunoreactivity was revealed by chemiluminescence using the ECL system.

\section{Histological examination}

Lung specimens were fixed in 10\% w/v PBS-buffered formaldehyde and embedded in paraffin. Histopathological examination was carried out on tissue sections, $7 \mu \mathrm{m}$ thick, stained with haematoxylin and eosin. 

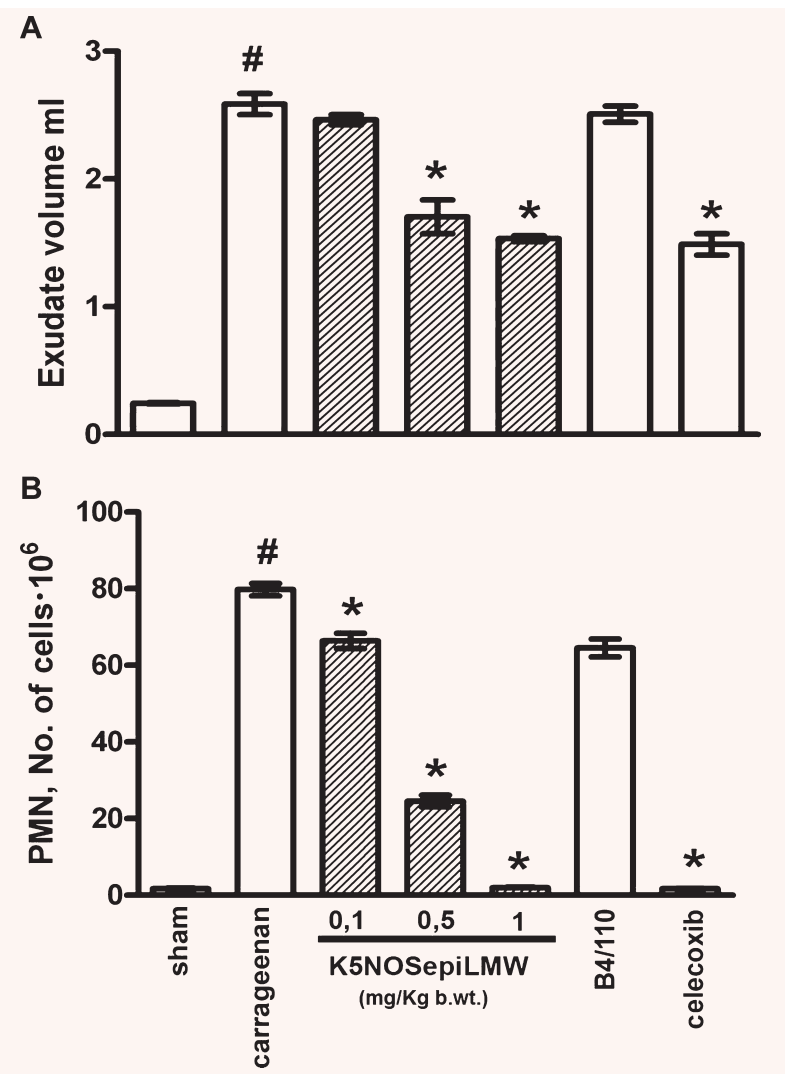

Fig. 1 Pleural exudate volume $(\mathbf{A})$ and number of polymorphonuclear cells, PMN, in the pleural fluid (B) in the different experimental groups, collected $4 \mathrm{hrs}$ after carrageenan injection. K5NOSepiLMW $(0.1-1 \mathrm{mg} / \mathrm{kg}$ b.wt.) and celecoxib (1 mg/kg b.wt.), but not compound B4/110 (1 mg/kg b.wt.), given 30 min. before carrageenan, significantly reduced these parameters as compared with carageenan alone. Significance of differences (one-way Anova, $n=8$ ): ${ }^{\#} P<0.001$ versus sham-operated controls; ${ }^{*} P<0.001$ versus carrageenan treated.

\section{Measurement of pro-inflammatory cytokines and PGE2}

Tumour necrosis factor (TNF)- $\alpha$ and IL-1 $\beta$ levels in the pleural exudate were evaluated using a commercial enzyme-linked immunosorbent assay kit (Calbiochem-Novabiochem, Milan, Italy) with a lower detection limit of $10 \mathrm{pg} / \mathrm{ml}$. PGE 2 levels were measured by radioimmunoassay (Amersham) without prior extraction, as described previously [26].

\section{Measurement of oxygen free radical scavenging properties of K5NOSepiLMW}

To evaluate whether the observed effects of K5NOSepiLMW could be attributable to intrinsic oxygen free radical scavenging properties, this substance was tested in a cell-free system using the xanthine-xanthine oxidase superoxide anion generating system [27]. Briefly, xanthine oxidase metab-

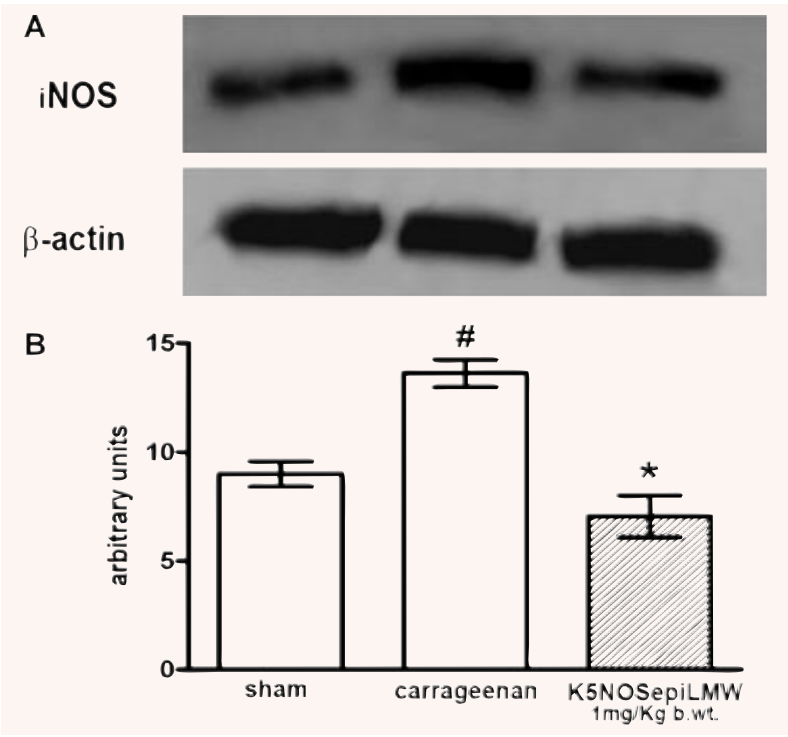

Fig. 2 iNOS protein expression evaluated by Western blot analysis in lung tissue samples from the different experimental groups, collected $4 \mathrm{hrs}$ after carrageenan injection. Carrageenan increases iNOS expression, whereas pre-treatment with K5NOSepiLMW (1 mg/kg b.wt.) inhibits this effect $(\mathbf{A})$. These data are confirmed by densitometric analysis (B). Significance of differences (one-way ANOVA, $n=8$ ): ${ }^{\#} P<0.001$ versus sham-operated controls; ${ }^{*} P<0.001$ versus carrageenan treated.

olizes in vitro its substrate xanthine and generates superoxide anion, which can be measured spectrophotometrically by the oxidation of the chromogen nitro blue tetrazolium (NBT) to formazan. K5NOSepiLMW was added to 10,100 and $1000 \mu \mathrm{g} / \mathrm{ml}$ (concentrations in the range of the dose administered to the rats) to a reaction solution containing $5 \mathrm{mU} / \mathrm{ml}$ xanthine oxidase, $250 \mu \mathrm{g} / \mathrm{ml}$ xanthine and $100 \mu \mathrm{M}$ NBT bromide in phosphate buffer, $\mathrm{pH} 7.4$. The samples were placed in a thermostat at $37^{\circ} \mathrm{C}$ and the reduction of NBT was evaluated spectrophotometrically at $570 \mathrm{~nm}$ wavelength after 5 and 15 min. of incubation.

\section{Statistical analysis}

Values are expressed as mean \pm S.E.M. Statistical significance of the differences between the experimental groups was checked by of one- or twoway ANOVA, as appropriate, followed by post hoc test for comparison between individual groups. A $P$-value $\leq 0.05$ was considered significant.

\section{Results}

All the rats treated with carrageenan developed an acute pleurisy characterized by the production of turbid exudate (Fig. 1A). Compared with the number of PMN collected from the pleural space of the sham-operated controls, injection of carrageenan induced a significant increase in the cell number (Fig. 1B). Pretreatments of rats with K5NOSepiLMW dose-dependently reduced 

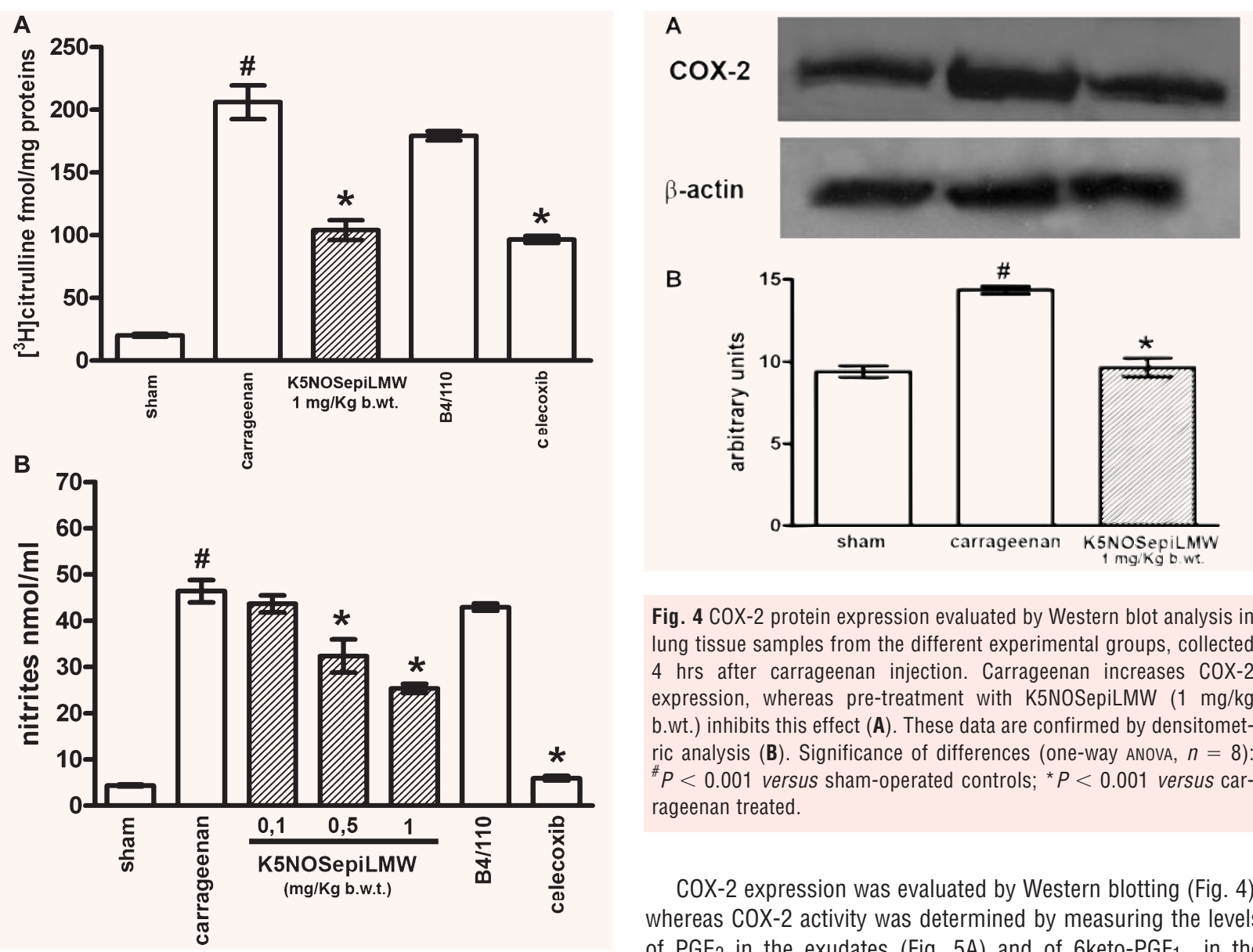

Fig. 3 iNOS enzyme activity, evaluated as L- $\left[{ }^{3} H\right]$ citrulline generation (A) and nitrite production (B), in lung tissue samples from the different experimental groups, collected 4 hrs after carrageenan injection. Carrageenan increases iNOS activity whereas pre-treatment with K5NOSepiLMW inhibits this effect (A, B). Moreover, K5NOSepiLMW (0.1-1 mg/kg b.wt.) and celecoxib ( $1 \mathrm{mg} / \mathrm{kg}$ b.wt.), but not compound B4/110 (1 mg/kg b.wt.), significantly reduce iNOS activity as compared with carageenan alone (A, B). Significance of differences (one-way AnOVA, $n=8$ ): ${ }^{\#} P<0.001$ versus sham-operated controls; ${ }^{*} P<0.001$ versus carrageenan treated.

the volume of the pleural exudate and the number of PMN within the exudate. These inhibitory effects were also observed when the rats were pre-treated with celecoxib (1 mg/kg b.wt.) but not with compound B4/110 (1 mg/kg b.wt.) (Fig. $1 A$ and B).

A significant increase in lung iNOS expression and activity was observed $4 \mathrm{hrs}$ after administration of carrageenan (Fig. 2A and B). Pre-treatment of rats with K5NOSepiLMW significantly reduced iNOS expression, evaluated by Western blot analysis (Fig. 2A), pre-treatment of rats with K5NOSepiLMW (1 mg/kg b.wt.) or celecoxib (1 mg/kg b.wt.) significantly reduced iNOS activity, measured as citrulline formation (Fig. 3A) and nitrite production (Fig. 3B). B4/110 (1 mg/kg b.wt.) did not cause any changes in iNOS activity (Fig. $3 A$ and B).

Fig. 4 COX-2 protein expression evaluated by Western blot analysis in lung tissue samples from the different experimental groups, collected 4 hrs after carrageenan injection. Carrageenan increases COX-2 expression, whereas pre-treatment with K5NOSepiLMW (1 mg/kg b.wt.) inhibits this effect $(\mathbf{A})$. These data are confirmed by densitometric analysis (B). Significance of differences (one-way ANOVA, $n=8$ ): ${ }^{\#} P<0.001$ versus sham-operated controls; ${ }^{\star} P<0.001$ versus carrageenan treated.

COX-2 expression was evaluated by Western blotting (Fig. 4), whereas COX-2 activity was determined by measuring the levels of $\mathrm{PGE}_{2}$ in the exudates (Fig. $5 \mathrm{~A}$ ) and of 6keto-PGF $\mathrm{F}_{1 \alpha}$ in the supernatant of lung tissue homogenates (Fig. 5B). In comparison with the sham-operated controls, the amount of COX-2 protein and the levels of both $\mathrm{PGE}_{2}$ and 6keto-PGF $\mathrm{P}_{\alpha}$ were greatly increased in the carrageenan-treated rats, an effect significantly and dose-dependently blunted by pre-treatment of the rat with K5NOSepiLMW and celecoxib (1 mg/kg b.wt.), but not with B4/110 (1 mg/kg b.wt.).

Carrageenan-treated rats exhibited a substantial increase in lung tissue MPO and TBARS, assumed as markers for PMN infiltration [24] and lipid peroxidation [25], respectively, as compared with the sham-operated controls (Fig. 6A and B). Pre-treatment of rats with K5NOSepiLMW attenuated in a dose-dependent fashion the increase in MPO and TBARS induced by carrageenan; celecoxib (1 mg/kg b.wt.) had a similar effect, while B4/110 (1 mg/kg b.wt.) was completely inactive (Fig. 6A and B).

Immunohistochemical analysis of lung sections obtained from carrageenan-treated rats revealed a positive staining for NT, which was absent in lung samples taken from the sham-operated controls (Fig. 7A and B). In contrast, no staining for NT was found in the lungs of carrageenan-treated rats that had been pre-treated with K5NOSepiLMW (1 mg/kg b.wt.) (Fig. 7C). Staining was absent in lungs from the sham-operated controls (not shown). 

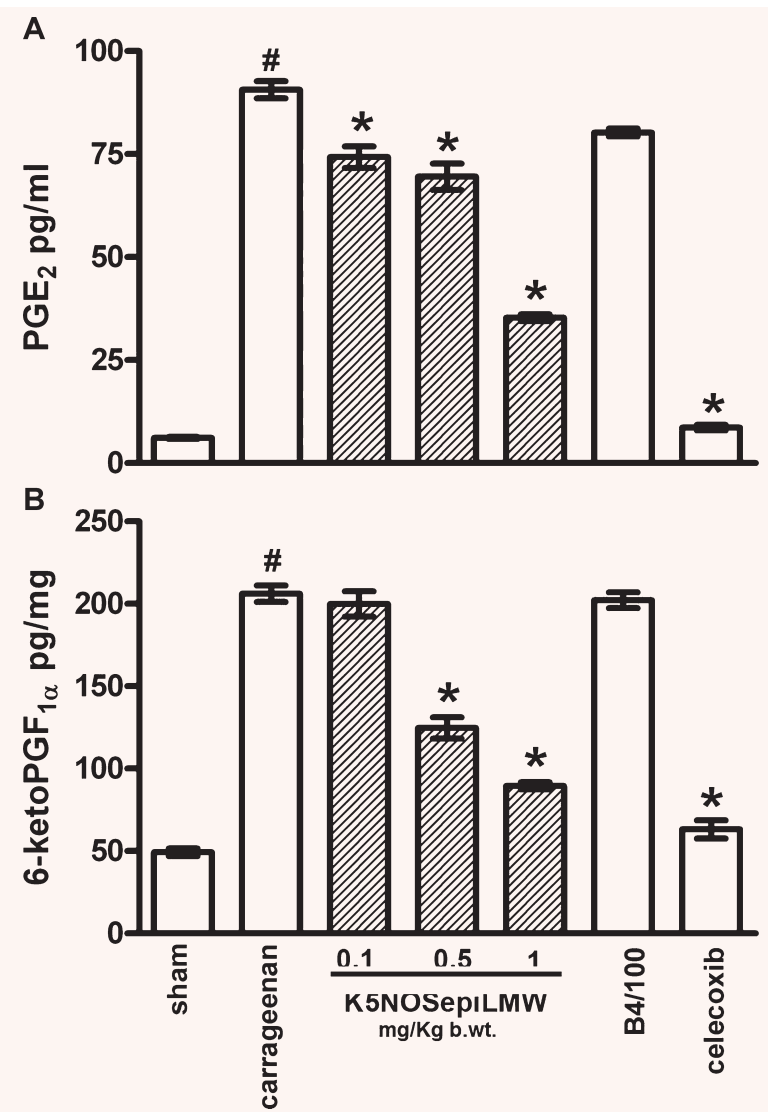

Fig. 5 COX-2 enzyme activity, evaluated as production of PGE2 (A) and 6keto-PGF1 $\alpha(\mathbf{B})$, in lung tissue samples from the different experimental groups, collected 4 hrs after carrageenan injection. Carrageenan increases COX-2 expression, whereas K5NOSepiLMW (0.1-1 mg/kg b.wt.) and celecoxib (1 $\mathrm{mg} / \mathrm{kg}$ b.wt.), but not compound B4/110 (1 mg/kg b.wt.), significantly reduced COX-2 activity as compared with carageenan alone (A, B). Significance of differences (one-way ANOVA, $n=8):{ }^{\#} P<0.001$ versus sham-operated controls; ${ }^{*} P<0.001$ versus carrageenan treated.

The immunohistochemical data was confirmed by dot blot analysis on lung tissue homogenates, showing that immunoreactive NT was almost undetectable in the sham-operated controls, was clearly present at high levels in the carrageenan-treated rats and became barely detectable upon pre-treatment with K5NOSepiLMW (1 mg/kg b.wt.) (Fig. 7D).

At variance with the normal histological appearance of the lungs from the sham-operated controls (Fig. 8A), the specimens from the rats treated with carrageenan showed tissue oedema, haemorrhage foci, infiltration with PMN and lymphocytes and reduced alveolar spaces (Fig. 8B). Pre-treatment K5NOSepiLMW (1 mg/kg b.wt.) reduced the above lung tissue abnormalities (Fig. $8 \mathrm{C}$ ).

The levels of the pro-inflammatory cytokines IL- $1 \beta$ and TNF- $\alpha$ were increased in the pleural exudate of the carrageenan-treated
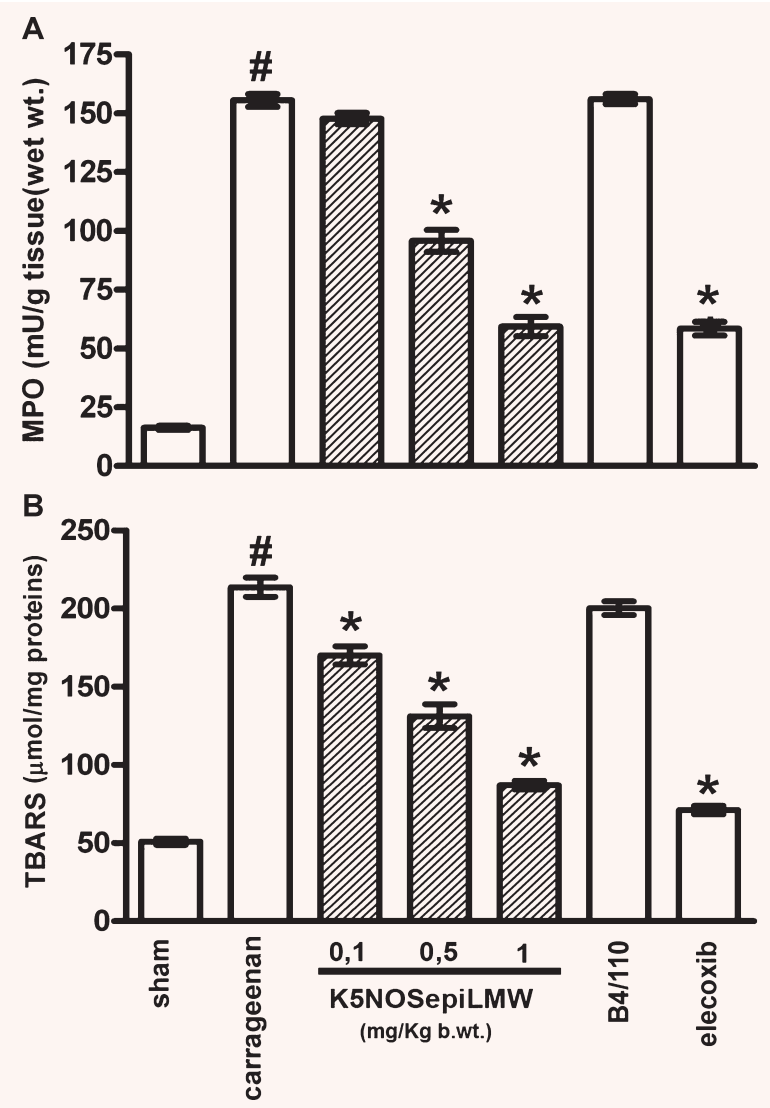

Fig. 6 Lung tissue myeloperoxidase, MPO, (A) and thiobarbituric acidreactive substances, TBARS, (B) in lung tissue samples from the different experimental groups, collected $4 \mathrm{hrs}$ after carrageenan injection. K5NOSepiLMW (0.1-1 mg/kg b.wt.) and celecoxib (1 mg/kg b.wt.), but not compound B4/110 (1 mg/kg b.wt.), significantly reduced these parameters as compared with carageenan alone. Significance of differences (one-way ANOVA, $n=8$ ): ${ }^{\#} P<0.001$ versus sham-operated controls; ${ }^{\star} P<0.001$ versus carrageenan treated.

rats compared with the sham-operated controls (Fig. 9A and B). The levels of both these cytokines were significantly and dosedependently reduced upon pre-treatment with K5NOSepiLMW and celecoxib (1 mg/kg b.wt.), but not with B4/110 (1 mg/kg b.wt.) (Fig. $9 \mathrm{~A}$ and $\mathrm{B}$ ).

K5NOSepiLMW, at any time-point tested, did not influence the levels of superoxide anion, expressed as $\mu \mathrm{mol}$ of formazan, generated by the xanthine-xanthine oxidase system, thus indicating that this molecule has no intrinsic oxygen free radical scavenging properties (5-min. incubation: control, $5.1+1.2$; K5NOSepiLMW: $10 \mu \mathrm{g} / \mathrm{ml}, 4.5+0.6 ; 100 \mu \mathrm{g} / \mathrm{ml}, 4.5+1 ; 1000 \mu \mathrm{g} / \mathrm{ml}, 3.2+0.3$; $P>0.05$. 15-min. incubation: control, $9.1+1.4$; K5NOSepiLMW: $10 \mu \mathrm{g} / \mathrm{ml}, 9.5+0.5 ; 100 \mu \mathrm{g} / \mathrm{ml}, 7.4+1.5 ; 1000 \mu \mathrm{g} / \mathrm{ml}, 6.1+$ $0.3 ; P>0.05)$. 


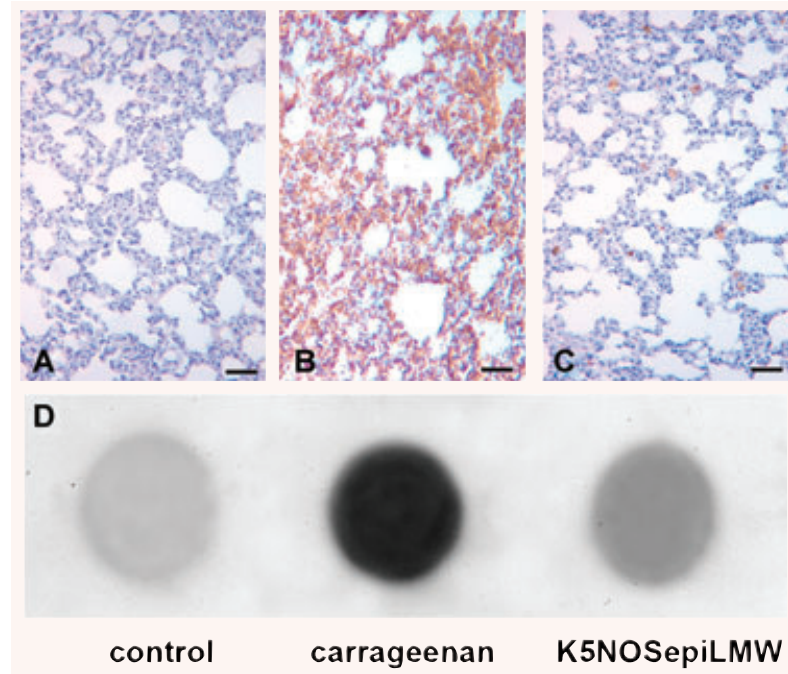

Fig. 7 Representative light micrographs of lung tissue immunostained to reveal nitrotyrosine (NT) in sham-operated rats $(\mathbf{A})$, carrageenan-treated rats (B) and rats given K5NOSepiLMW (1 mg/kg b.wt.) $30 \mathrm{~min}$. before carrageenan (C). Carrageenan causes the appearance of a clear-cut NT immunostaining, whereas K5NOSepiLMW nearly abolishes this effect. Bars $=50 \mu \mathrm{m}$. Dot blot analysis on lung tissue homogenates (D) shows that carrageenan treatment increases the amount of NT-containing proteins, whereas pre-treatment with K5NOSepiLMW inhibits this effect.

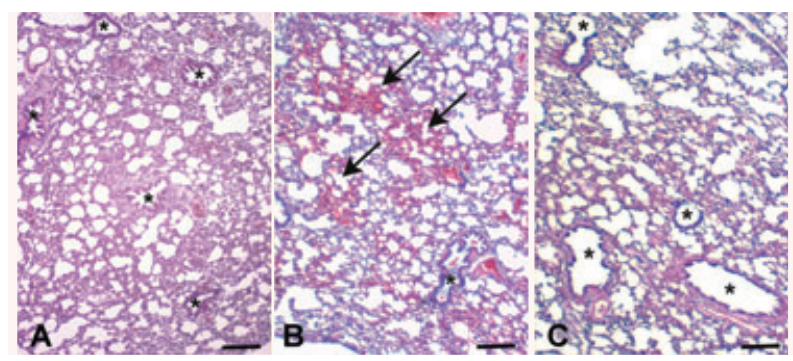

Fig. 8 Representative light micrographs of lung tissue in sham-operated controls $(\mathbf{A})$, carrageenan-treated rats $(\mathbf{B})$ and rats given K5NOSepiLMW (1 mg/kg b.wt.) $30 \mathrm{~min}$. before carrageenan (C). Carrageenan causes the appearance of tissue oedema, haemorrhage foci, leucocyte infiltration and reduction of alveolar spaces (arrows), whereas K5NOSepiLMW blunts these histopathological changes. Asterisks label bronchial lumina. Bars $=100 \mu \mathrm{m}$.

\section{Discussion}

This study provides evidence that a low molecular weight semisynthetic 0-sulphated heparin derivative (K5NOSepiLMW) markedly reduces the acute inflammatory response of carrageenaninduced pleurisy in the rat in vivo, a commonly used animal model to evaluate the efficacy of non-steroidal anti-inflammatory drugs (NSAIDs). A 30-min pre-treatment with K5NOSepiLMW, injected

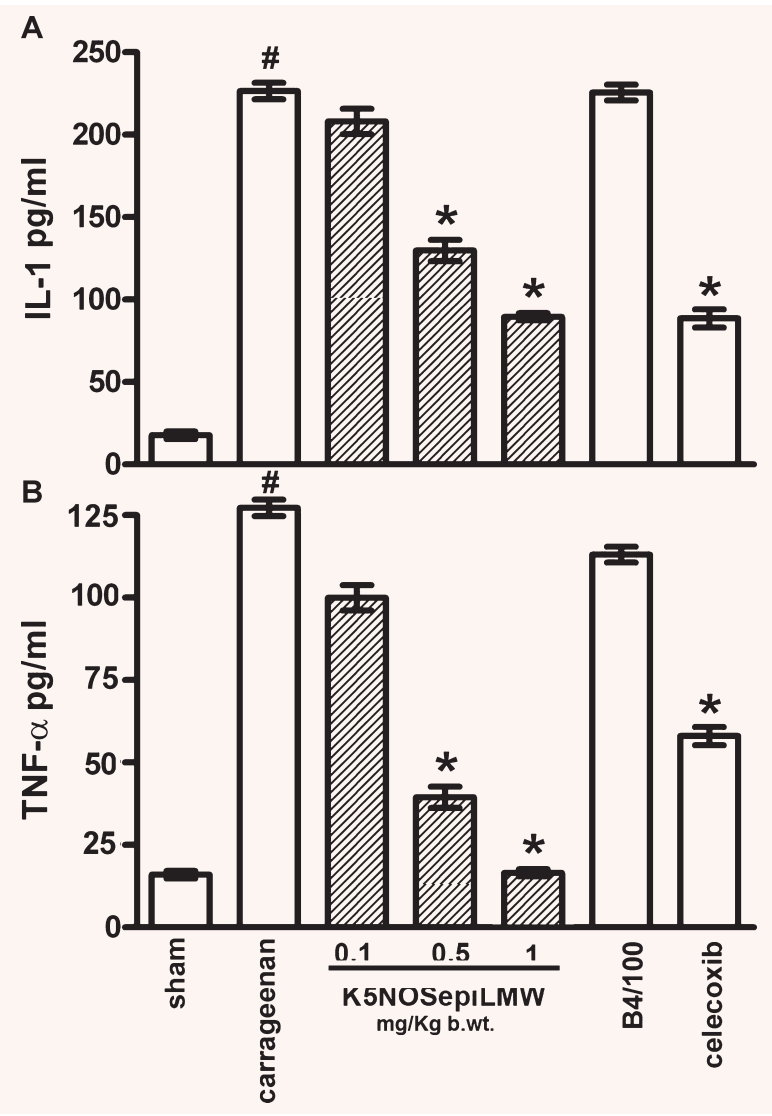

Fig. 9 Levels of IL-1 $\beta(\mathbf{A})$ and TNF- $\alpha(\mathbf{B})$ in the pleural exudates collected from the different experimental groups, collected 4 hrs after carrageenan injection.. K5NOSepiLMW (0.1-1 mg/kg b.wt.) and celecoxib $(1 \mathrm{mg} / \mathrm{kg}$ b.wt.), but not compound B4/110 (1 mg/kg b.wt.), significantly reduced the amounts of these pro-inflammatory cytokines as compared with carageenan alone. Significance of differences (one-way ANOVA, $n=8$ ): ${ }^{\#} P<0.001$ versus sham-operated controls; ${ }^{*} P<0.001$ versus carrageenan treated.

locally at doses of $0.1,0.5$ and $1 \mathrm{mg} / \mathrm{kg} \mathrm{b.wt.,} \mathrm{attenuated} \mathrm{the}$ recruitment of inflammatory leucocytes in the lung tissue and the pleural exudate, inhibited the induction of iNOS and COX-2, thereby abating the generation of harmful nitric oxide and proinflammatory PGs, such as $\mathrm{PgE}_{2}$ and $\mathrm{PGF}_{1 \alpha}[16,28]$, reduced the inflammation-induced nitroxidative stress, as shown by TBARS and NT, and blunted the local generation of cytokines such as IL-1 $\beta$ and TNF- $\alpha$. As expected, all these parameters were markedly up-regulated by intrapleural injection of carrageenan in the absence of any pre-treatment. The anti-inflammatory effects of K5NOSepiLMW appear specific, as judged by the marked doseresponse relationship in the $0.1-1 \mathrm{mg} / \mathrm{kg}$ b.wt. range; in this respect, K5NOSepiLMW is fairly potent, as its effects correspond to $1 \mathrm{mg} / \mathrm{kg}$ b.wt. of celecoxib, a selective COX-2 inhibitor assumed as reference NSAID. It is conceivable that the observed effect of K5NOSepiLMW to inhibit COX-2 and PG generation may be due, 
at least in part, to inhibition of iNOS activity and nitric oxide production, as nitric oxide can activate COX-2 activity [29]. On the other hand, the current findings allow ruling out that the observed protection from lung inflammation afforded by K5NOSepiLMW may be related to oxygen free radical scavenging properties, as this molecule was unable to decrease the levels of superoxide anion generated by an in vitro free radical generating system, such as xanthine-xanthine oxidase.

The current findings are in keeping with previous studies showing that low molecular weight heparins are capable of functionally inhibiting the cells of acute and chronic inflammatory response, decreasing PMN and monocyte chemotaxis and activation [30-32] and inhibiting fibroblast proliferation, endothelial activation and neo-angiogenesis [33-35]. The low molecular weight of K5NOSepi is a useful feature for the anti-inflammatory activity of this substance, as it may allow this molecule to enter the target cells where it exerts its anti-inflammatory effects by interfering with the expression of pro-inflammatory cytokines [14]. This assumption is in keeping with previous reports that low molecular weight heparins and non-anticoagulant heparins blunt pro-inflammatory cytokine generation by inhibiting nuclear translocation of the transcription factor NF-кB [32, 36], although we have no direct evidence that such mechanism of action is also operating in K5NOSepiLMW. As IL-1 $\beta$ and TNF- $\alpha$ are known to mediate the induction of iNOS and COX-2 [37, 38], it is conceivable that the reduced levels of these inducible pro-inflammatory enzymes observed in the present study may be secondary to a reduced formation of endogenous $\mathrm{IL}-1 \beta$ and TNF- $\alpha$ by K5NOSepiLMW. The possibility that K5NOSepiLMW could directly inhibit iNOS and COX-2 activity cannot be ruled out, but the present findings do not allow clarifying this issue. Moreover, the marked anti-inflammatory properties of K5NOSepiLMW appear to be related to 0 -sulphation, which enable it to bind to and inactivate endothelial $\mathrm{L}$ - and $\mathrm{P}$-selectins, thus counteracting leucocyte rolling and adhesion [13].

In conclusion, this study provides support to the concept that semi-synthetic, low molecular weight heparin derivatives may represent a novel class of potent NSAIDs which, compared with unfractionated full-sized heparins, lack pro-angiogenic properties and substantial effects on the coagulation system [39]. Based on its specific pharmacological effects highlighted in the present study, K5NOSepiLMW could be suitable as an endothelial protecting drug for the treatment of vascular inflammation, potentially useful for the prevention of cardiovascular disease, which encompasses hypertension, atherothrombosis, myocardial infarction, stroke, peripheral vascular disease and renal failure.

\section{Acknowledgement}

This investigation was supported by a grant from the Ente Cassa di Risparmio di Firenze, Florence, Italy.

\section{References}

1. Xie WL, Chipman JG, Robertson DL, et al. Expression of a mitogen-responsive gene encoding prostaglandin synthase is regulated by mRNA splicing. Proc Natl Acad Sci USA. 1991; 88: 2692-6.

2. Sluiter W, Pietersma A, Lamers JMJ, et al. Leukocyte adhesion molecules on the vascular endothelium: their role in the pathogenesis of cardiovascular disease and the mechanism underlying their expression. J Cardiovasc Pharmacol. 1993; 22: S37-44.

3. Laroux FS, Lefer DJ, Kawachi S, et al. Role of nitric oxide in the regulation of acute and chronic inflammation. Antiox Redox Signal. 2000; 2: 391-6.

4. Johnson KJ, Varani J, Smolen JE. Neutrophil activation and function in health and disease. Immunol Ser. 1992; 57: 1-46.

5. Feuerstein G, Hallenbeck JM. Prostaglandins, leukotrienes, and platelet-activating factor in shock. Annu Rev Pharmacol Toxicol. 1987; 27: 301-13.

6. Tomlinson A, Appleton I, Moore AR, et al. Cyclo-oxygenase and nitric oxide synthase isoforms in rat carrageenin- induced pleurisy. Br J Pharmacol. 1994; 113: 693-8.

7. Guyton JR, Rosenberg RD, Clowes AW, et al. Inhibition of rat arterial smooth muscle cell proliferation by heparin. In vivo studies with anticoagulant and nonanticoagulant heparin. Circ Res. 1980; 46: 625-34.

8. Folkman J. Regulation of angiogenesis: a new function of heparin. Biochem Pharmacol. 1985; 34: 905-9.

9. Au YP, Montgomery KF, Clowes AW. Heparin inhibits collagenase gene expression mediated by phorbol ester-responsive element in primate arterial smooth muscle cells. Circ Res. 1992; 70: 1062-9.

10. Weiler JM, Edens RE, Linhardt RJ, et al. Heparin and modified heparin inhibit complement activation in vivo. J Immunol. 1992; 148: 3210-5

11. Nelson RM, Cecconi 0, Roberts WG, et al. Heparin oligosaccharides bind $\mathrm{L}-$ and $\mathrm{P}$-selectin and inhibit acute inflammation. Blood. 1993; 82: 3253-8.

12. Attanasio M, Gori AM, Giusti B, et al. Cytokine gene expression in human LPS- and IFNgamma-stimulated mononuclear cells is inhibited by heparin. Thromb Haemost. 1998; 79: 959-62.

13. Wang JG, Mu JS, Zhu HS, et al. $\mathrm{N}$-desulfated non-anticoagulant heparin inhibits leukocyte adhesion and transmigration in vitro and attenuates acute peritonitis and ischemia and reperfusion injury in vivo. Inflamm Res. 2002; 51: 435-43.

14. Gori AM, Attanasio M, Gazzini A, et al. Cytokine gene expression and production by human LPS-stimulated mononuclear cells are inhibited by sulfated heparin-like semi-synthetic derivatives. J Thromb Haemost. 2004; 2: 1657-62.

15. Gilroy DW, Colville-Nash PR, Willis D, et al. Inducible cyclooxygenase may have anti-inflammatory properties. Nat Med. 1999; 5: 698-701.

16. Cuzzocrea S, Mazzon E, Calabro G, et al. Inducible nitric oxide synthase-knockout mice exhibit resistance to pleurisy and lung injury caused by carrageenan. $A m \mathrm{~J}$ Respir Crit Care Med. 2000; 162: 1859-66. 
17. Cuzzocrea S, Santagati S, Sautebin L, et al. 17beta-estradiol antiinflammatory activity in carrageenan-induced pleurisy. Endocrinology. 2000; 141: 1455-63.

18. Cuzzocrea S, Mazzon E, Sautebin L, et al. The protective role of endogenous estrogens in carrageenan-induced lung injury in the rat. Mol Med. 2001; 7: 478-87.

19. Cuzzocrea S, Mazzon E, Sautebin L, et al. Protective effects of Celecoxib on lung injury and red blood cells modification induced by carrageenan in the rat. Biochem Pharmacol. 2002; 63: 785-95.

20. Cuzzocrea S, McDonald MC, Mazzon E, et al. Effects of 5-aminoisoquinolinone, a water-soluble, potent inhibitor of the activity of poly (ADP-ribose) polymerase, in a rodent model of lung injury. Biochem Pharmacol. 2002; 63: 293-304.

21. Cuzzocrea S, Zingarelli B, Hake $P$, et al. Antiinflammatory effects of mercaptoethylguanidine, a combined inhibitor of nitric oxide synthase and peroxynitrite scavenger, in carrageenan-induced models of inflammation. Free Radic Bio Med. 1998; 24: 450-9.

22. Tomlinson A, Appleton I, Moore AR, et al. Cyclo-oxygenase and nitric oxide synthase isoforms in rat carrageenin-induced pleurisy. Br J Pharmacol. 1994; 113: 693-8.

23. Bradford MM. A rapid and sensitive method for the quantitation of microgram quantities of protein utilizing the principle of protein-dye binding. Anal Biochem. 1976; 72: 248-54.

24. Mullane KM, Kraemer R, Smith B. Myeloperoxidase activity as a quantitative assessment of neutrophil infiltration into ischemic myocardium. I Pharmacol Methods. 1985; 14: 157-67.

25. Ohkawa H, Ohishi N, Yagi K. Assay for lipid peroxides in animal tissues by thiobarbituric acid reaction. Anal Biochem. 1979; 95: 351-8.

26. Sautebin $\mathrm{L}$, Ialenti $\mathrm{A}$, lanaro $\mathrm{A}$, et al. Endogenous nitric oxide increases prostaglandin biosynthesis in carrageenin rat paw oedema. Eur J Pharmacol. 1995; 286: 219-22.

27. Goldstein IM, Kaplan HB, Edelson HS, et al. Ceruloplasmin: an acute phase reactant that scavenges oxygen-derived free radicals. Ann N Y Acad Sci. 1982; 389: 368-79.

28. Harada Y, Hatanaka K, Kawamura M, et al. Role of prostaglandin $\mathrm{H}$ synthase-2 in prostaglandin E2 formation in rat carrageenin-induced pleurisy. Prostaglandins. 1996; 51: 19-33.

29. Salvemini D, Misko TP, Masferrer JL, et al. Nitric oxide activates cyclooxygenase enzymes. Proc Natl Acad Sci U S A. 1993; 90: 7240-4.

30. Matzner Y, Marx G, Drexler R, et al. The inhibitory effect of heparin and related glycosaminoglycans on neutrophil chemotaxis. Thromb Haemost. 1984; 52: 134-7.

31. Tangelder GJ, Arfors KE. Inhibition of leukocyte rolling in venules by protamine and sulfated polysaccharides. Blood. 1991; 77: 1565-71.

32. Hochart H, Jenkins PV, Smith OP, et al. Low-molecular weight and unfractionated heparins induce a downregulation of inflammation: decreased levels of proinflammatory cytokines and nuclear factorkappaB in LPS-stimulated human monocytes. Br J Haematol. 2006; 133: 62-7.

33. Bârzu T, Lormeau JC, Petitou M, et al. Heparin-derived oligosaccharides: affinity for acidic fibroblast growth factor and effect on its growth-promoting activity for human endothelial cells. J Cell Physiol. 1989; 140: 538-48.

34. Deepa PR, Varalakshmi P. Favourable modulation of the inflammatory changes in hypercholesterolemic atherogenesis by a low-molecular-weight heparin derivative. Int J Cardiol. 2006; 106: 338-47.

35. Norrby K. Low-molecular-weight heparins and angiogenesis. Acta Pathol Microbiol Immunol Scand. 2006; 114: 79-102.

36. Thourani VH, Brar SS, Kennedy TP, et al. Nonanticoagulant heparin inhibits NFkappaB activation and attenuates myocardial reperfusion injury. Am J Physiol Heart Circ Physiol. 2000; 278: H2084-93.

37. Szabo C, Wu CC, Gross SS, et al. Interleukin-1 contributes to the induction of nitric oxide synthase by endotoxin in vivo. Eur J Pharmacol. 1993; 250: 157-60.

38. Akarasereenont P, Bakhle YS, Thiemermann C, et al. Cytokine-mediated induction of cyclo-oxygenase-2 by activation of tyrosine kinase in bovine endothelial cells stimulated by bacterial lipopolysaccharide. Br J Pharmacol. 1995; 115: 401-8.

39. Schulman S. Novel anticoagulant agents: introduction. J Intern Med. 2003; 254: 308-12. 